\title{
DEPROJECTION OF PLANETARY NEBULA IMAGES
}

\author{
K. VOLK and D. A. LEAHY \\ Department of Physics and Astronomy, University of Calgary, 2500, University Drive \\ N.W., Calgary, Alberta, Canada T2N 1 N4
}

We present first results of the deprojection of some optical and radio images of planetary nebulae using an iterative technique based on the Lucy method, as discussed in Leahy (1991). This approach allows a greater range of possible geometries to be investigated and is less sensitive to the effects of noise compared with the direct matrix-inversion method which can be used for the spherical case. We assume that the emissivity function can be represented as

$$
\epsilon(r, \theta)=f(r) g(\theta)
$$

where $(\mathbf{r}, \theta)$ are polar coordinates in a plane defined by our line of sight to the nebula and a radial cut of the sky image $(\theta=0$ in the plain of the sky). By analyzing a number of cuts at different orientations we can approximate the three-dimensional emissivity function. In these models we assume that $g(\theta)$ is of the form $\cos ^{\mathrm{N}}\left(\theta-\theta_{0}\right)$ with $\mathrm{N}=0$ (symmetric case) or $\mathrm{N}=2$, and derive $f(r)$. So far we have always found that $\mathrm{N}=2$ gives a much better result than $\mathrm{N}=0$, which often cannot give any reasonable fit to the data. The $\theta_{0}$ parameter allows for a tilt of the plane of symmetry of the nebula with respect to the plane of the sky. So far we have done only a few trials with $\theta_{0} \neq 0$.

The initial results suggest that the studied nebulae (NGC 40, NGC 6720, M3-35, NGC 6790, and IC 5117) have incomplete interacting-winds shells covering of order $25 \%$ of the sky as viewed from the star, and would appear more or less bi-polar if viewed in the plane of the sky. At intermediate orientations the nebulae would generally appear as incomplete rings. In some directions it appears that either no interacting-wind shell has formed or the shell has been disrupted. We suggest that the mass-loss process while the stars were on the AGB were quite asymmetric, so that when the second, fast wind develops the interacting-winds bubble quickly blows out and thereafter the sections of the shell which remain enter a snow-plow phase. Our results indicate that the nebulae may be better described in cylindrical coordinates $(r, \phi, z)$ with considerable $\phi$ dependence of the structure. We hope to develop a version of the deprojection program which uses cylindrical coordinates to investigate this possibility.

\section{References}

Leahy, D. A. 1991, A\&A, 247, 584 